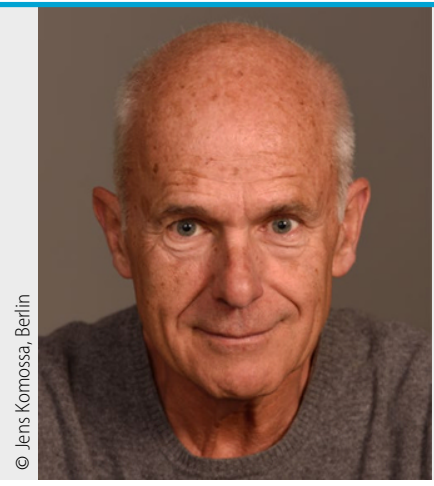

॥) Wir brauchen die Impfungen, um die schwerwiegenden

neurologischen Folgen der SARS-CoV-2(,severe acute

respiratory syndrome coronavirus 2")-Infektion und des

Post-COVID-Syndroms zu verhindern

Prof. Dr. Peter Berlit

Deutsche Gesellschaft für Neurologie (DGN)

Reinhardtstr. 27C, 10117 Berlin, Deutschland

berlit@dgn.org

\title{
COVID-19 („,coronavirus disease 2019“) und kein Ende?
}

Ende dieses Jahres haben wir fast 2 Jahre der Coronapandemie hinter uns, 2 Jahre, in denen sich für uns privat, aber auch im Gesundheitswesen vieles geändert hat, auch und insbesondere für die Neurologie. Nachdem 2020 zunächst nur in Kasuistiken über neurologische Manifestationen der SARS-CoV-2-Infektion berichtet worden war, wurde mit der Zeit klar, dass Enzephalopathien, Schlaganfälle, Enzephalomyelitiden und verschiedene Formen neuromuskulärer Manifestationen zu dem Krankheitsbild gehören. In einem Cochrane-Review waren Anosmie und Ageusie die einzigen Symptome der SARS-CoV-2-Infektion, die mit einer „red flag“ versehen waren, also als pathognomonisch gelten [1].

Wir haben hunderte von Arbeiten gescannt, gelesen und bewertet, um mit dem DGN-Journal Club Sie, unsere Mitglieder, auf dem Laufenden zu halten. Und wenn wir ehrlich sind: Die Quantität der Publikationen ist riesig, die Qualität lässt hingegen oft zu wünschen übrig.

Dies macht auch die Entwicklung von Leitlinien zu COVID-19 zu einer schwierigen Aufgabe. Zurzeit sind wir dabei, unsere eigene Leitlinie $\mathrm{zu}$ den neurologischen Manifestationen als „living guideline“ von S1 auf S2 upzugraden. Die Leitlinie zu Long- und Post-COVID ist auf S1-Niveau und die zur Rehabilitation bei COVID auf S2-Niveau veröffentlicht.

Immerhin gibt es eine S3-Leitlinie, nämlich zur stationären Behandlung von COVID-19 (AWMF-Registernummer [AWMF: Arbeitsgemeinschaft der Wissenschaftlichen Medizinischen Fachgesellschaften e.V.] 113-001). Während hier die Indikationen für eine stationäre Aufnahme und die Einleitung einer Beatmung bzw. einer Intensivtherapie relativ klar formuliert werden können, fehlen nach wie vor wirklich gute medikamentöse Therapieoptionen. Nach zahlreichen offenen Studien gibt es allerdings inzwischen eine ganze Reihe randomisiert kontrollierter Untersuchungen (RCT) sowie inzwischen auch Metaanalysen. Die klarste Empfehlung kann ausgesprochen werden für das uns Neurologen wohl bekannte Dexamethason auf der Basis von 6 RCT mit insgesamt 7595 Patientinnen und Patienten sowie ei-

DGNeurologie 2021 • 4 (6): 433-434

https://doi.org/10.1007/s42451-021-00387-7

Angenommen: 17. September 2021

(c) Springer Medizin Verlag GmbH, ein Teil von Springer Nature 2021 ner Metaanalyse. Eine moderate Empfehlung kann abgegeben werden für Janus-Kinase(JAK)-Inhibitoren und Tocilizumab. Und wir wissen inzwischen auch, dass alle stationär behandelten Patientinnen und Patienten mit COVID-19 eine prophylaktische niedrig dosierte Antikoagulation erhalten sollten und dass Risikopatientinnen und -patienten auch von einer höher dosierten Antikoagulation profitieren.

Bei widersprüchlichen Ergebnissen kann für bzw. gegen Remdesivir keine klare Empfehlung ausgesprochen werden. Aber zum Glück ist inzwischen klar, welche Medikamente nicht wirken. Hierzu zählen Chloroquin, Colchicin, Azithromycin, Ivermectin, Vitamin D, Anakinra und Lopinavir/Ritonavir. Wenn bedacht wird, dass für alle diese Substanzen zunächst positive offene Studien publiziert wurden und zahlreiche Politiker einige dieser Medikamente zu Beginn der Pandemie als Wunderwaffen deklarierten, sind wir durchaus einen Schritt weiter. Aber insgesamt bleiben unsere therapeutischen Möglichkeiten bei der SARS-CoV-2-Infektion doch sehr begrenzt.

Umso wichtiger ist es, die Erkrankung zu verhindern. Impfungen sind der einzige Weg, die SARS-CoV-2-Pandemie zu beenden. Das Virus wird weiter Mutationen ausbilden, die eine raschere Verbreitung und stärkere Schädigung des Organismus ermöglichen. Nur durch wiederholtes und möglichst vollständiges Impfen wird es möglich sein, die Infektionsketten zu durchbrechen und die z. T. schwerwiegenden neurologischen Folgen der SARS-CoV-2-Infektion zu verhindern. Die zur Verfügung stehenden Impfstoffe sind hochwirksam, können aber über die häufigeren leichten grippeähnlichen Nebenwirkungen hinaus in Einzelfällen auch zu schweren neurologischen Komplikationen führen. Hierzu zählen das Guillain-Barré-Syndrom (GBS), Enzephalomyelitiden sowie Hirnvenenthrombosen. Allerdings sind alle diese neurologischen Komplikationen während und nach der SARS-CoV-2-Infektion um ein Vielfaches häufiger als nach der Impfung, und im Unterschied zur COVID-19-Infektion können wir die Impfnebenwirkungen erfolgreich behandeln.

Das impfassoziierte GBS spricht auf i.v. Immunglobuline (IVIG) an. Multiple Sklerose(MS)-ähnliche Symptome wie Sehnerv- oder Rückenmarksentzündung respondieren auf die hochdosierte Gabe von Kortikoiden. Diese Beobachtung und die typischen MRT- (Magnetresonanztomographie), Liquor- und Antikörperbefunde sprechen im Übrigen dafür, dass es sich bei diesen impfassoziierten Ereignissen vermutlich oft um die Erst- 
manifestation einer MS bzw. einer Neuromyelitis-optica-Spektrum-Erkrankung (NMOSD) in zeitlichem Zusammenhang mit der Impfung handelt, dass also keine Kausalität vorliegt.

Bei der vakzininduzierten immunogenen thrombotischen Thrombozytopenie (VITT) nach den Vektorimpfstoffen von AstraZeneca und Johnson \& Johnson ist dies anders. Hierbei treten aufgrund eines spezifischen autoimmunologischen Mechanismus innerhalb der ersten 3 Wochen nach der Impfung Hirnvenenthrombosen, Pfortaderthrombosen, Lungenembolien, Blutungen und Schlaganfälle auf. Ursächlich sind Antikörper gegen den Komplex aus Heparin und Plättchenfaktor 4 (PF4), welche bei dieser Impfkomplikation zu einer Thrombozytopenie führen. Da die PF4-Antikörper gleichzeitig die übrigen Thrombozyten aktivieren, treten Thrombosen bei gleichzeitiger Blutungsneigung wegen des Plättchenmangels auf. $\mathrm{Zu}$ den Labortests für die Diagnose gehören vordringlich die Bestimmung der Thrombozytenzahl und der D-Dimere sowie gezielt die Suche nach Antikörpern gegen den Plättchenfaktor 4 (PF4) mittels ELISA (enzymverknüpftes immunbindendes Nachweisverfahren [„,enzyme linked immunosorbent assay“], kein andersartiger HIT-Suchtest! [HIT: heparininduzierte Thrombozytopenie]), mit einem Plättchenaktivierungstest zur Bestätigung. Zur Behandlung werden erfolgreich hochdosiert IVIG, kombiniert mit einer Antikoagulation, eingesetzt [2].

Laut einer Fallserie der Charité-Universitätsmedizin Berlin lassen sich bei Menschen, die sich nach Impfung mit dem AstraZeneca-Vakzin wegen heftiger Kopfschmerzen vorstellen und alle Laborkriterien einer VITT erfüllen, durch eine sofort eingeleitete Behandlung Hirn- oder Sinusvenenthrombosen und andere vaskuläre Komplikationen verhindern. Offensichtlich bietet das Prä-VITT-Syndrom ein therapeutisches Fenster, um den Impffolgen effektiv entgegenzuwirken [3]. Damit können wir die neurologischen Komplikationen der SARS-CoV-2-Impfungen behandeln, und wir benötigen diese, um die schwerwiegenden neurologischen Folgen der SARS-CoV-2-Infektion und des Post-COVID-Syndroms zu verhindern.

Wenn bedacht wird, dass regelmäßige Grippeimpfungen über eine Aktivierung der Mikroglia mit Clearing von Betaamyloid das Demenzrisiko reduzieren können [4], ist es durchaus denkbar, dass auch die SARS-CoV-2-Impfungen zu solchen positiven neurologischen Langzeiteffekten führen können. So könnte die Impfung nicht nur Post-COVID-Syndrome verhindern, sondern womöglich auch einer Neurodegeneration vorbeugen. Wir haben also mehr als einen Grund, unseren Patientinnen und Patienten zur SARS-CoV-2-Impfung zu raten!

Interessenkonflikt. P. Berlit gibt an, dass kein Interessenkonflikt besteht.

Literatur

1. Struyf T, Deeks JJ, Dinnes J et al (2021) Cochrane COVID-19 Diagnostic Test Accuracy Group. Signs and symptoms to determine if a patient presenting in primary care or hospital outpatient settings has COVID-19. Cochrane Database Syst Rev. https://doi.org/10.1002/14651858.CD013665.pub2

2. Greinacher A, Thiele T, Warkentin TE et al (2021) Thrombotic Thrombocytopenia after ChAdOx1 nCov-19 Vaccination. N Engl J Med 384(22):2092-2101. https:// doi.org/10.1056/NEJMoa2104840

3. Salih F, Schönborn L, Kohler S et al (2021) Vaccine-induced thrombocytopenia with severe headache. N Engl J Med. https://doi.org/10.1056/NEJMc2112974
4. Wiemken TL, Salas J, Hoft DF et al (2021) Dementia risk following influenza vaccination in a large veteran cohort. Vaccine 39(39):5524-5531. https:// doi.org/10.1016/j.vaccine.2021.08.046 